\title{
On the constant domains principle and its weakened versions in the Kripke sheaf semantics
}

\author{
Dmitrij Skvortsov* \\ All-Russian Institute of Scientific and Technical Information (VINITI), \\ Molodogvardejskaja 22, korp.3, kv.29, Moscow, RUSSIA, 121351 \\ skvortsovd@yandex.ru
}

We consider superintuitionistic predicate logics understood in the usual way, as sets of predicate formulas (without function symbols) containing all axioms of Heyting predicate logic Q-H and closed under modus ponens, generalization, and substitution of arbitrary formulas for atomic ones (we are mainly interested in logics without equality, and only sometimes we mention logics with equality).

1 We consider the semantics of predicate Kripke frames with equality (called $I$-frames, for short), which is equivalent to the semantics of Kripke sheaves (see [3]). Namely, a (predicate) Kripke frame is a pair $M=(W, U)$ formed by a poset $W$ with the least element $0_{W}$ and a domain map $U$ defined on $W$ such that $U(u) \subseteq U(v)$ for $u \leq v$. An $I$-frame is a triple $M=(W, U, I)$, in which $(W, U)$ is a Kripke frame and $I$ is a family of equivalence relations $I_{u}$ on $U(u)$ for $u \in W$ such that $I_{u} \subseteq I_{v}$ for $u \leq v$.

A valuation $u \vDash A$ (for $u \in W$ and formulas $A$ with parameters replaced by elements of $U(u)$ ) satisfies the monotonicity: $u \leq v, u \vDash A \Rightarrow v \vDash A$

and the usual inductive clauses for connectives and quantifiers, e.g.

$u \vDash(B \rightarrow C) \Leftrightarrow \forall v \geq u[(v \vDash B) \Rightarrow(v \vDash C)]$,

$u \vDash \forall x B(x) \Leftrightarrow \forall v \geq u \forall c \in U(v)[v \vDash B(c)]$,

etc. (for the case with equality, $a=b$ is interpreted by $a I_{u} b$ in an $I$-frame and by $a=b$ in a usual Kripke frame, for $a, b \in U(u)$ ). For an $I$-frame we admit only the valuations preserving $I_{u}$ (on every $U(u), u \in W)$, i.e.,

$$
\bigwedge_{i}\left(a_{i} I_{u} b_{i}\right) \Rightarrow\left(u \vDash A\left(a_{1}, \ldots, a_{n}\right) \Leftrightarrow u \vDash A\left(b_{1}, \ldots, b_{n}\right)\right) .
$$

A formula $A(\mathbf{x})$ is valid in $M$ if it is true under any valuation in $M$, i.e., if $u \vDash A(\mathbf{a})$ for any $u \in W$ and $\mathbf{a} \in\left(D_{u}\right)^{n}$. The predicate logic $\mathbf{L}(M)$ of an (I-)frame $M$ is the set of all formulas valid in $M$.

We consider the constant domains principle

$$
D=\forall x(P(x) \vee Q) \rightarrow \forall x P(x) \vee Q
$$

(where $P$ and $Q$ are unary and 0 -ary symbols, respectively), and two its weakened versions, namely:

$$
\begin{gathered}
D^{-}=\forall x(\neg P(x) \vee Q) \rightarrow \forall x \neg P(x) \vee Q, \quad \text { and } \\
D^{*}=\forall x(P(x) \vee Q) \rightarrow Q \vee \forall x \exists y(P(y) \& \neg \neg[R(x, x) \rightarrow R(x, y)]) ;
\end{gathered}
$$

here $D^{*}$ simulates the following formula with equality:

$$
D_{=}^{*}=\forall x(P(x) \vee Q) \rightarrow Q \vee \forall x \exists y(P(y) \& \neg \neg[x=y])
$$

*The work on this paper was supported by the Russian Foundation for Basic Research (project no. 11-01-93107) 
(i.e., $\left.\left[\mathbf{Q}-\mathbf{H}^{=}+D^{*}\right]=\left[\mathbf{Q}-\mathbf{H}^{=}+D_{=}^{*}\right]\right)$. The formula $D$ states (in an $\left(I\right.$-)frame) that $U(u)=U\left(0_{W}\right)$ for every $u \in W$, i.e., $\forall a \in U(u)\left[a \in U\left(0_{W}\right)\right]$. Now, $D^{*}$ states that any individual $a \in U(u)$ has a $\neg \neg$-ancestor in $0_{W}$,

i.e., there exists $b \in U\left(0_{W}\right)$ such that $u \vDash \neg \neg(a=b)$.

Similarly, $D^{-}$states that $\forall a \in U(u) \exists b \in U\left(0_{W}\right)[u \not \forall \neg(a=b)]$.

Clearly, $D \vdash D^{*} \vdash D^{-}$(we write $A \vdash B$ for $[\mathbf{Q}-\mathbf{H}+A] \vdash B$ ).

The logic $\left[\mathbf{Q}-\mathbf{H}+D^{-}\right]$is incomplete w.r.t. $I$-frames (as well as some its natural extensions); its completion (which is not finitely axiomatizable) is described in [5. On the other hand, 7 ] proved the completeness w.r.t. $I$-frames (i.e., the Kripke sheaf completeness) for the following logics: $\left[\mathbf{Q}-\mathbf{H}+D^{*}\right],\left[\mathbf{Q}-\mathbf{H}+D^{*} \& K\right],\left[\mathbf{Q}-\mathbf{H}+D^{*} \& K \& J\right]$, and the incompleteness for $\left[\mathbf{Q}-\mathbf{H}+D^{*} \& J\right]$,
where $K=\neg \neg \forall x(P(x) \vee \neg P(x)) \quad$ (Kuroda's formula)
and $\quad J=\neg Q \vee \neg \neg Q \quad$ (weak excluded middle).

This shows that in some sense the axiom $D^{*}$ for the Kripke sheaf semantics behaves like the axiom $D$ for the usual Kripke semantics; recall that the logics $[\mathbf{Q}-\mathbf{H}+D],[\mathbf{Q}-\mathbf{H}+D \& K]$, and $[\mathbf{Q}-\mathbf{H}+D \& K \& J]$ are Kripke-complete, while $[\mathbf{Q}-\mathbf{H}+D \& J]$ is Kripke-incomplete.

And here we claim that this similarity is not too close.

Recall that $\left[\mathbf{H}+P_{2} \& J\right]$ is the greatest non-classical superintuitionistic propositional logic (here $P_{2}=\left[Q_{0} \vee\left(Q_{0} \rightarrow Q \vee \neg Q\right)\right]$ is the axiom of height 2).

Lemma (Main Lemma). [Q-H $\left.+P_{2} \& J+D^{*}\right] \not \forall K$.

On the other hand, clearly, $K$ is valid in every $(I$-)frame of a finite height. Therefore,

Theorem. Let $[\mathbf{Q}-\mathbf{H}+\mathbf{\Lambda}] \subseteq \mathbf{L} \subseteq\left[\mathbf{Q}-\mathbf{H}+P_{2} \& J+D^{*}\right]$ for a superintuitionistic propositional $\operatorname{logic} \boldsymbol{\Lambda}$ of a finite slice. Then $\mathbf{L}$ is Kripke sheaf incomplete.

Corollary. The logics $\left[\mathbf{Q}-\mathbf{H}+\boldsymbol{\Lambda}+D^{*}\right]$ and $\left[\mathbf{Q}-\mathbf{H}+\boldsymbol{\Lambda}+D^{-}\right]$are Kripke sheaf incomplete for every non-classical superintuitionistic propositional $\operatorname{logic} \boldsymbol{\Lambda}$ of a finite slice.

Recall that the logics $[\mathbf{Q}-\mathbf{H}+\boldsymbol{\Lambda}+D]$ are Kripke-complete e.g. for all $\boldsymbol{\Lambda}$ being tabular logics or subframe logics (i.e., logics axiomatizable by $\rightarrow$-formulas) (see [4, Theorems 3.7 and 3.9]), while the logics $[\mathbf{Q}-\mathbf{H}+\boldsymbol{\Lambda}]$ are Kripke-incomplete for all non-classical $\boldsymbol{\Lambda}$ of finite slices [2, Theorem 3.2] (and moreover, they are Kripke sheaf incomplete as well, see [3, a Remark in Sect. 9]).

By the way, note that the logics $\left[\mathbf{Q}-\mathbf{H}+\boldsymbol{\Lambda}+D^{*}\right]$ and $\left[\mathbf{Q}-\mathbf{H}+\boldsymbol{\Lambda}+D^{-}\right]$are different for every non-classical $\boldsymbol{\Lambda}$, since it was claimed in [6] that

$$
\left[\mathbf{Q}-\mathbf{H}+P_{2} \& J+D^{-}\right] \nvdash D^{*} .
$$

Also it was shown in [6] that every $I$-frame (i.e., every Kripke sheaf) validating $D^{-} \& J$ validates $D^{*}$ as well. Here we claim even more:

Proposition. Let $\boldsymbol{\Lambda}$ be a superintuitionistic propositional logic. Then:

(every $I$-frame validating $\left[\mathbf{Q}-\mathbf{H}+\boldsymbol{\Lambda}+D^{-}\right]$validates $D^{*}$ ) iff $\boldsymbol{\Lambda} \vdash \delta J$.

Here $\quad \delta J=\left[Q_{0} \vee\left(Q_{0} \rightarrow \neg Q \vee \neg \neg Q\right)\right]$

(this formula states that $J$ holds in all strictly future worlds: 'tomorrow $J$ ').

2 The proof of Main Lemma uses the functor semantics, see [1].

Namely, let $\mathcal{C}$ be a category with a frame representation $W$; this means that $W=O b(\mathcal{C})$ is the set of objects of $\mathcal{C}$ pre-ordered by the following relation: 
$u \leq v$ iff $\mathcal{C}(u, v) \neq \varnothing$, i.e., iff in $\mathcal{C}$ there exists a morphism from $u$ to $v$.

A $\mathcal{C}$-set (a SET-valued functor, or a presheaf over $\mathcal{C}$, inhabited, i.e., with non-emptiness assumption) is a triple $\mathbb{F}=(W, \bar{D}, \bar{E})$, in which $\bar{D}=\left(D_{u}: u \in W\right)$ is a family of disjoint non-empty domains and $\bar{E}=\left(E_{\mu}: \mu \in \operatorname{Mor}(\mathcal{C})\right)$ is a family of functions with $E_{\mu}: D_{u} \rightarrow D_{v}$ whenever $\mu \in \mathcal{C}(u, v)$ (i.e., $\mu$ is a morphism from $u$ to $v$ ), satisfying the usual requirements:

$$
\begin{aligned}
E_{\mu \circ \mu^{\prime}}= & E_{\mu^{\prime}} \circ E_{\mu} \quad \text { for } \mu \in \mathcal{C}(u, v), \mu^{\prime} \in \mathcal{C}(v, w) \\
& \text { (i.e., } \left.E_{\mu \circ \mu^{\prime}}(a)=E_{\mu^{\prime}}\left(E_{\mu}(a)\right) \text { for any } a \in D_{u}\right), \quad \text { and }
\end{aligned}
$$

$E_{1_{u}}=1_{D_{u}} \quad$ (the identity function on $D_{u}$ corresponds to the identical morphism $\left.1_{u} \in \mathcal{C}(u, u), u \in W\right)$.

A valuation $u \vDash A(\mathbf{a})$ (for $u \in W$ and $\left.\mathbf{a}=\left(a_{1}, \ldots, a_{n}\right) \in\left(D_{u}\right)^{n}\right)$ in $\mathbb{F}$ satisfies the monotonicity:

$$
u \vDash A(\mathbf{a}) \Rightarrow v \vDash A\left(E_{\mu}(\mathbf{a})\right) \quad \text { for } u \leq v \text { and } \mu \in \mathcal{C}(u, v),
$$

and the usual inductive clauses for connectives and quantifiers, e.g. $u \vDash(B \rightarrow C)(\mathbf{a}) \Leftrightarrow \forall v \geq u \forall \mu \in \mathcal{C}(u, v)\left[v \vDash B\left(E_{\mu}(\mathbf{a})\right) \Rightarrow v \vDash C\left(E_{\mu}(\mathbf{a})\right]\right.$, $u \vDash \forall x B(\mathbf{a}, x) \Leftrightarrow \forall v \geq u \forall \mu \in \mathcal{C}(u, v) \forall c \in D_{v}\left[v \vDash B\left(E_{\mu}(\mathbf{a}), c\right)\right]$, etc. (here we write $E_{\mu}(\mathbf{a})=\left(E_{\mu}\left(a_{1}\right), \ldots, E_{\mu}\left(a_{n}\right)\right)$ for $\left.\mathbf{a}=\left(a_{1}, \ldots, a_{n}\right)\right)$.

A formula $A(\mathbf{x})$ is valid in a $\mathcal{C}$-set $\mathbb{F}$ if it is true w.r.t. all valuations in $\mathbb{F}$, i.e., if $u \vDash A(\mathbf{a})$ for all $u \in W$ and $\mathbf{a} \in\left(D_{u}\right)^{n}$. The predicate logic of a $\mathcal{C}$-set $\mathbb{F}$ is the set

$\mathbf{L}(\mathbb{F})=\{A \mid$ all substitution instances of $A$ are valid in $\mathbb{F}\} ;$

note that the set of formulas valid in $\mathbb{F}$ in general is not substitution closed (cf. e.g. [3] Remark in Sect. 5]).

Lemma. Let $\mathcal{C}$ be a category with one object 0 and two arrows: $1_{0}$ and $\mu_{0}$.

Let $\mathbb{F}$ be a $\mathcal{C}$-set with two-element domain $D_{0}=\{a, b\}, E_{\mu_{0}}(a)=E_{\mu_{0}}(b)=b$.

Then $P_{2} \& J \& D^{*} \in \mathbf{L}(\mathbb{F})$, and $K \notin \mathbf{L}(\mathbb{F})$.

To conclude, let us mention the following well-known

Fact. Let $W_{0}$ be a (rooted) poset. Then the following conditions are equivalent:

(1) $K \in \mathbf{L}(M)$ for every Kripke frame $M$ based on $W_{0}$,

(2) $K \in \mathbf{L}(M)$ for every $I$-frame $M$ based on $W_{0}$, and

(3) $W_{0}$ satisfies the McKinsey property: $\forall u \exists v \geq u\left[v\right.$ is maximal in $\left.W_{0}\right]$.

On the other hand, $K$ is valid e.g. in every frame $M$ with a finite constant domain (based on an arbitrary $W$ ).

Now we can give a counterpart to this fact for the functor semantics:

Claim. Let $\mathcal{C}_{0}$ be a category. Then the following conditions are equivalent:

(1) $K \in \mathbf{L}(\mathbb{F})$ for every $\mathcal{C}_{0}$-set $\mathbb{F}$, and

(2) $\forall u \exists v \geq u[\forall w \geq v(v \geq w)]$ (i.e., $v$ lies in a maximal cluster of the frame representation $W_{0}$ of $\left.\mathcal{C}_{0}\right)$ and

for every $v$ in a maximal cluster of $W_{0}: \forall \mu \in \mathcal{C}(v, v) \exists \mu^{\prime} \in \mathcal{C}(v, v)\left[\mu \circ \mu^{\prime}=1_{v}\right]$.

Note that every frame $M=(W, U)$ (with a finite constant domain) can be represented as an isomorphic $\mathcal{C}$-set (for any category $\mathcal{C}$ based on $W$ ).

\section{References}

[1] S. Ghilardi. Presheaf semantics and independence results for some non-classical first-order logics. Archive for Math. Logic, 29:125-136, 1989. 
[2] H. Ono. Model extension theorem and Craig's interpolation theorem for intermediate predicate logics. Reports on Math. Logic, 15:41-58, 1983.

[3] V. Shehtman and D. Skvortsov. Semantics of non-classical first order predicate logics. In P. Petkov, editor, Mathematical Logic, pages 105-116, N.Y., 1990. Plenum Press. (Proc. of Summer school and conference in mathematical logic 'Heyting' 88 ').

[4] T. Shimura. Kripke completeness of some intermediate predicate logics with the axiom of constant domain and a variant of canonical formulas. Studia Logica, 52:23-40, 1993.

[5] D. Skvortsov. The Kripke sheaf completion of superintuitionistic predicate logic with a weak version of the axiom of constant domain. In preparation.

[6] D. Skvortsov. On the predicate logic of linear Kripke frames and some its extensions. Studia Logica, 81:261-282, 2005.

[7] D. Skvortsov. Kripke sheaf completeness of some superintuitionistic predicate logics with a weakened constant domains principle. Studia Logica, 100:361-383, 2012. 